\title{
A SURVEY ON SMART HOME AUTOMATION
}

\author{
Shauvik Purkayastha \\ Dept. of CSE \\ Assam Engineering College \\ Guwahati, India
}

\author{
Triporna Kashyap \\ Dept. of CSE \\ Assam Engineering College \\ Guwahati, India
}

\author{
Navam Pradhan \\ Dept. of CSE \\ Assam Engineering College \\ Guwahati, India
}

\begin{abstract}
The human race has always been in the quest for a better and easier life and ever since technology began heavily integrating itself into the society. From the appliances in our kitchen to the phones in our palms, there are many high tech devices that make our lives easier. But it would be more convenient if we could control these devices remotely. An automated system could control machines and perform tasks as per the convenience of the user from long distances. In this paper, we are presenting a proposed system for a Smart Home automation where the user can control home electronic appliances easily and with higher controllability using an android application. To design this system, we are using a Raspberry Pi module. The Raspberry Pi acts as a mini computer.
\end{abstract}

$\begin{array}{lcrr}\begin{array}{l}\text { Keywords-Smart } \\ \text { Raspberry }\end{array} \quad \begin{array}{c}\text { Home } \\ \text { Pi, }\end{array} & \begin{array}{c}\text { automation, } \\ \text { Android }\end{array} \\ \text { Electric/electronic appliances } & \end{array}$

\section{INTRODUCTION}

Home automation system or a Smart Home refers to an automated system whose application varies from basic remote controlling of lighting to complex computer or micro-controller based networks involving varying degrees of intelligence and automation. It cannot just control home appliances but also cater to the entertainment needs of the user. The user can benefit from a home automation system due to convenience it provides, energy saving nature, and safety benefits leading to improved quality of life. Most of the technology that we are using today sprouts from ideas in popular science fictions. The best science fiction has an uncanny knack for predicting future developments in technology. Throughout history, bizarre and eccentric concepts that once seemed chimerical are now a part of daily life and a common sight in the average home. Similarly, the initial smart homes were not actual structures but ideas. Science fiction has explored the idea of home automation for decades. Prolific writers, such as Ray Bradbury, imagined a future where homes were interactive, and apparently ran themselves. In the novel Demon Seed (1973) penned by Dean Koontz, the author writes about an entire home operated by a computer program. Alfred, the program, controlled everything from temperature to lighting, security and even cooking.

In this project we are using a Raspberry $\mathrm{Pi}$ microcontroller. We have done so because the microcontroller can perform only one operation at a time. Performing two tasks simultaneously is complex. A computer could hence be used but it is not cost effective and consumes lot of power. The best alternative is a Raspberry Pi which is like a mini computer. The idea of controlling objects remotely is very fascinating and beneficial. The motive behind the goal is very simple that is, it is not always possible to be near to the home physically but it's very important to control the appliances for many purposes like saving energy, cost etc. So the automation system takes the control of the home. It would serve mankind well and make lives more safe and comfortable.

\section{RELATED WORKS}

Here we will discuss about the various papers that we have been able to collect regarding our topic. Below are the various papers that we have studied and their reviews.

In [1], this paper presents an application of Raspberry Pi in home automation control through the Internet (E-mail) where the subject of the received email is read by the python coded algorithm which is fed into Raspberry Pi and system responds to these corresponding instructions. The devices to be controlled are connected to the Pi through a relay is driven circuit. This is done because of the different power ratings of the appliances and the Raspberry Pi. The code was first set to load and log into the home g-mail account by using the email library of python IDE. After successful initialization, the Pi starts reading the subjects of emails from the account specified in the code. The subject of these e-mails is 


\section{International Journal of Engineering Applied Sciences and Technology, 2019 \\ Vol. 4, Issue 3, ISSN No. 2455-2143, Pages 496-501 \\ Published Online July 2019 in IJEAST (http://www.ijeast.com)}

then compared with the initializing commands of the associated devices and the control signal is generated according to it on the corresponding GPIO pin. This whole process repeated after every 0.5 seconds.

This system is interactive, efficient and flexible according to the consumer needs. It immediately replies about the status of work done by Raspberry Pi to the consumer. The proposed system has been experimented practically employing LEDs as switching signal indicators.

In [2], Here they have made a wireless remote control system for controlling lights and fan via $\mathrm{Wi}-\mathrm{Fi}$ capable handheld devices such as Smartphone. They have used Raspberry $\mathrm{Pi}$ as the MCU that will communicate with the devices which takes the instructions from over the internet given by the mobile phone.

In [3], Here they struggle the observation and dominant of the device via WWW through a program through an internet login portal. They will communicate with a home automation network through an online entree, by suggests that of low power communication protocols like ZigBee, Wi-Fi, etc. This project aims at dominant home appliances via Smartphone victimization. $\mathrm{Wi}-\mathrm{Fi}$ as a communication protocol and Raspberry $\mathrm{Pi}$ as a server system. The user here will traverse directly with the system by moving through a web-based interface over the online, whereas home appliances like lights, fan and door lock are remotely controlled through the simple web site. An extra feature that enhances the aspect of protection from fire accidents is its capability of police work the smoke so as that intervals the event of any fire, it associates Associate in Nursing alert message at the side of a picture that is shipped to the good mobile device of the user. The server is going to be interfaced with hardware circuits (using relays) that management appliances running reception. The communication with the server permits the user to pick out the acceptable device. The communication with the server permits the user to choose out the suitable device. The server communicates with the corresponding relays. If the online association is down or the server isn't up, the embedded system board will still manage and operate the appliances domestically while not an online association.

In this paper [4], Girish Birajdar and Shrikant Mahindrakar have developed a way to automate home appliances using an Embedded Webserver combined with a Raspberry Pi. Here they have used a Raspberry Pi microcontroller, a digiduino board, electromagnetic relay and Weaved services. The main focus of the paper was on controlling devices remotely. They have connected the GPIO pins of the Raspberry Pi to the electromagnetic relay but since it cannot provide enough voltage the digiduino board acts as an intermediary between the two. The digiduino board boosts the $3.3 \mathrm{~V}$ received from the $\mathrm{Pi}$ to the $5 \mathrm{~V}$ required by the relay. Their work is different from other notable works in the aspect that they have extended the remote controlling from local area network to the Internet. The major problem lies in access of the Raspberry Pi from the Internet is that it cannot be controlled by a public IP address and needs a static IP address. To do this, Weaved cloud services is used. The task of Weaved cloud services is to bind the public IP address of the Raspberry Pi with the static IP address provided by the service. With the help of this static IP address, we can gain access to the WebTop framework of buttons and when a button is pushed, the signal is sent to the Raspberry Pi through the Internet with the help of cloud service. This is transmitted to the Local Area Network of Raspberry pi triggering the GPIO pins.

In [5], This paper focuses on building smart home automation where the user can control the home appliances using a mobile phone or a laptop with Internet connectivity. The current status of the devices (whether ON or OFF) is acquired along with the live stream from the camera of the Raspberry Pi. This data is then updated to the database of the website. The website displays the current status of the devices and also the live stream on a monitor when connected through the HDMI port. For this purpose, they have used a Raspberry Pi which is using the Advanced Reduced Instruction Set Computing Machine (ARM) technology. They have used Simple Computer Vision Techniques (CVT) to detect the occurrence of any movement. In case of motion detection, the user is alerted by means of an SMS using the GSM module connected to the Raspberry Pi. They have also used the MJPG-streamer. It is a command line tool to stream JPEG files over an IPbased network from the webcam to a viewer like Firefox, Video LAN or even to a Windows Mobile gadget using the TCPMP-Player. In comparison to others, this particular software was used because it is faster than most of the streamers. Due to its speed, it is ideal for remote control projects which require real-time video feed for navigation and orientation purposes.

In [6], Here they have used an I2C connection between Raspberrypi2 and Arduino Uno where the Pi acts as the master and the Arduino UNO board acts as the slave. They have used firebase cloud by 
Google for exchange of data between the Android and pi. They have focused mainly on 5 areas. Air Flow regulation, Automatic Gas Removal, Garden Maintenance, Rain water Harvester, and Security. The sensors used are Passive Infrared Motion sensor (PIR), Temperature and Humidity sensor (DHT11), Light Sensor (LDR). Soil Moisture sensor, Magnetic Reed sensor, Raindrop sensor (LM393), Gas Sensor (MQ5). These sensors continuously monitor the environment in the home and relay them to the Arduino which sends them to the Pi for processing. The Pi Web server takes care of the entire network management and database maintenance. It issues the requests to the cloud to send push notifications and react to the user input from the Android application.

In [7], The work done in this paper focuses on remotely controlling home appliances. But since a multitude of projects is done in this arena, this project aims to design a cheap, open source and easy to use the system. To control the web appliance remotely they have designed an HTML and PHP based webpage. The Raspberry Pi can be made to act as a web server to host the web page by using an Apache server. PHP is used for communication or sending/receiving messages between the user and the web interface as well as between the web page and the Pi module. A DS18B20 temperature sensor is used to monitor temperature and other security zones. The temperature gets updated on the web page each time the page loads. This also includes a power switch which allows remote monitoring and controlling the lights of the house through a mobile device and reduces the human load of operating them manually. These peripherals are controlled via a webbased interface served on the Raspberry Pi and accessible from inside or outside the home.

In [8], IoT involves enhancing the network to proficiently collect and analyze the info from varied sensors and actuators then sends the info to the transportable or a private pc over a wireless association. The aim of this technique is to supply security and police investigation to a house through the net. During this paper, the planned system is intended using ARM-11 design and UNIX operating system OS based mostly Raspberry Pi-3 board, DC motor, and USB camera. The DC motor is connected to theRaspberryPi-3 board via driving circuit (L293D) to manage the door whereas the camera is interfaced with the USB port of Raspberry Pi-3 board. an internet page is provided to the tip user with username and countersign so as to allow the entry of solely licensed users. When with success work in, the user is ready to manage the door by exploitation the open and shut buttons and is ready to look at the live streaming video of the required location that is that the door surroundings. A capture button is additionally provided so as to require snaps of the running video.

In this project, a digital camera is connected to 1 of the USB ports on the Raspberry Pi board. A DC motor is connected to GPIO pins of the Raspberry Pi through a driver circuit for dominant of the door. a specific IP-address is assigned by the router that is interfaced with the Raspberry Pi through associate coax. Upon startup, the Raspberry Pi posts the login type i.e., index.html on its assigned IP-address by default, an individual with username and countersign should be connected to an equivalent router so as to envision the result. when authentication, a replacement page pops-up containing the info from the camera i.e., live streaming and additionally OPEN, shut and CAPTURE buttons for dominant the door and taking footage. Hence, a system which might monitor the house at the door or the other place of interest is developed.

In this paper, varied ideas of UNIX operating system, Python, IoT, hypertext markup language and Raspberry Pi has been incorporated so as to with success implement the system. The system is appropriate for the period of time home safety observation and for remotely dominant the door lockup system. The system is also utilized in several places like banks, hospitals, labs, offices, etc that dramatically crop the hazard of unauthorized entry.

In [9], The work done in this project proposes the development of a personal assistant that helps users interact with household appliances using speech and gesture commands to provide a more interactive and user friendly living experience. It also incorporates the integration of various tools and components developed during the execution of the project.

The focus of this work is to help users to operate home appliances with speech or gesture commands which give the user a better sense of control. The work also aims at building an inexpensive personal assistant which is achieved using a Raspberry Pi that used Google's Speech Recognition application interface (API) to convert spoken text, picked up using a microphone to written text (Python strings). To implement the above goals, the following methodology is followed:

- Understanding the dynamics of each part of the system 


\section{International Journal of Engineering Applied Sciences and Technology, 2019 \\ Vol. 4, Issue 3, ISSN No. 2455-2143, Pages 496-501 \\ Published Online July 2019 in IJEAST (http://www.ijeast.com)}

- Determination of required project hardware components

- Implement smart control of residential lighting - A smart alarm system synced to email.

This paper [10] aims to propose a security door lock system based on Raspberry Pi technology where cameras, keypad, and pi-lids are being utilized to produce an associate frightful system that has the ability to advise the owner, as well as, recognizing guests by giving them a user id. during this system, the approved individuals are solely those UN agencies that can get permission to access the doors. The system works by taking snaps of the guest through a code and camera pi positioned within the doors then, these snaps are sent to the owner of the house.

A smart digital door lock is a system to monitor and control several devices in the home. Our smart digital door lock system operates over internet network by using Raspberry Pi3Input phase aims to input the key by a newcomer and if the key is valid or the image of the newcomer is matching with the image in the database, the door is opened, else the door is not opened and a photo is taken and sent to the owner of the home by-mail and the owner of the house wishes to all to enter, the key and the image of the person is stored in the database. The input to the control module is key pad of the user or visitor. The control module then starts the camera PI capture image and sends the file to the database through Email. The output basically includes access to the door for the user at the door by using LEDs (red and green).

The keypad is the input module when the guest inserts the code at the door. It then sends a signal to control module" Raspberry pi3" which then check the guest id by the database. The control module then sends the guest-id to the server for verification. The server then searches for guest-id and if the id exists in the database then the server sends a signal back to control module as a response and at the same time activates the camera and take snaps of the user at the door and sends it through Email.

The system uses face recognition technique using the Fisher method to compare the image with the existing database and by this process, the visitor is allowed to enter or not to enter. This report tries to propose a door access and monitoring control system which consist of different stages:

- Detecting by keypad and camera pi user • Fetching user id

- Verification

- Information

- Process according to request.

In this paper [11] a worldwide accessible Cloudbased automation system is presented and implemented using Raspberry Pi. The proposed system can be used in industrial sectors to reduce the waste of electric power, and providing notification in case of fault or abnormal disturbances in the industry. This system consists of two major units: the first one is the Web Server or Cloud where user can locally and remotely monitor the current status of the residential/industrial machinery and sensors. The proposed solution can be applied in industries where the status of machinery can be observed and operated through this system. In addition, it can be used in residential areas; for example, if someone left this/her home or working places and forget to switch off his/her electrical appliances, still, he/she can check and control the status of his/her home appliances through the web while staying outside.

The main objective of this work is to implement a complete control system which can be applied to control home appliances, offices and machines of industries from any remote location through Cloud using Raspberry Pi. It starts with User Login panel to authenticate user access where user can log in with their Email address and Password. If the user's Email address and Password match with the user's details stored in the database, then he/she can proceed to the Project Dashboard or maintenance panel. After Logging in through user login panel, users can see the Dashboard where all the sensor's outputs are fetched from a table of the database using PHP functions.

In this work [12] describes an experimental implementation of smart home automation using Raspberry Pi, Facebook Chatbot and Google Maps APIs.

The design of smart home automation system in this project was targeted at its simplicity, nonintrusiveness, and user-friendliness. Any typical user would prefer to use software with familiar User Interface (UI), send simple instructions/commands.

With this intention in mind, chatbot (Chat robot or sometimes merely bot) was selected for this purpose to help provide nice UI. enrich users' experiences for their smart home automation. Facebook's official launch of its chatbot API (Application Program Interface) for Messenger is officially named "Messenger Platform" will allow us to create a simple yet versatile chatbot for home automation. The objective of this project is to develop the chatbot on Facebook to serve as a point of contact to control the home appliances over the Internet. Additional features like a calculation of the duration of the user's current location to his/her home by using Google Maps APIs. It chiefly includes of 2 
major components, namely, cloud and residential service. To utilize home automation during this system, a user should be the one WHO initiates the speech with the Chatbot through Facebook chat service. Whenever our larva receives the message, the Facebook server can convey that message to the cloud server through services provided by Heroku, wherever each knowledge and information process is programmed to figure hand and glove as per style. 3 main options of this larva are: Case one Turn on - the device can activate. Case a pair of Turn off the device can put off. Case three Home -the larva can respond with a matter to raise user location. Standing of the output to Raspberry $\mathrm{Pi}$ is keep during this information at Heroku server for retrieval. to attain this, Heroku has got to analyze the message sent from a user through web hook with proper authentication from Facebook to the Heroku server and then update the state of the home appliance in the database accordingly. Smart home automation via Facebook Chatbot has been proposed to all ow users to access and take control of their home appliances remotely and literally from anywhere.

All these papers helped us to get a better understanding of the topic. They helped us in devising a methodology for our project. Since we already knew the advantages, disadvantages, limitations of various Smart Home building techniques through these papers, we are able to choose a simple yet effective method for our project. The next chapter discusses in detail the methodology taken for the project.

\section{PROPOSED MODEL}

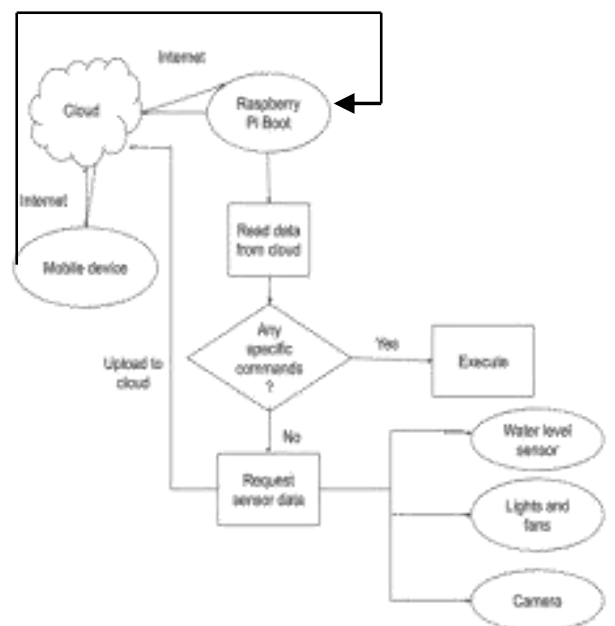

Fig. 1. Proposed Model
The purpose of our project is to design smart home automation for the user. By using this smart home automation, the user can control home appliances like lights, fans, water pumps and so on using an Android application. The user can also request for images of the surroundings in case of an emergency. These images will be sent to the e-mail account of the user. This chapter delves into the methodology employed by us in building the project, the designing of the Android app and the scripts running on the Raspberry Pi. Before starting our project we first drew up a rough plan of how we were going to proceed with our work. We designed a flow chart to be followed during the entire duration of work. We have used the ThingSpeak Cloud API to send and receive signals from the Raspberry Pi. The sensors- HC-SR04 Ultrasonic sensor and the Pi Camera sensors are connected to the Raspberry Pi. The Pi receives the sensor data from these sensors and uploads them to the cloud. An app will be designed so that the user can control the electronic devices, get alert notifications etc. The user canal so receive status updates of all the devices connected to the Pi.

\section{CONCLUSION}

Smart home automation exploitation raspberry pi is associate in nursing application of the internet of things. During this paper, a epitome good home automation exploitation IoT is bestowed. These works are carried forward by integration relays to the Raspberry Pi board for dominant home appliances from an overseas location in a very real state of affairs. Expected to grow in quality within the close to future is that the use of good home merchandise to extend family safety, specifically associated with fireplace protection and carbon monoxide gas observance. Momentarily we tend to connect and dominant solely some devices in home appliances however within the future, we are going to be connecting multiple devices so as to regulate it from everywhere the globe.

\section{REFERENCES}

[1] J. Sarthak, V. Anant and G. Lovely, February 2014, "Raspberry Pi based Interactive Home Automation System through E-mail”, International Conference on Reliability, Optimization and Information Technology (ICROIT). 


\section{International Journal of Engineering Applied Sciences and Technology, 2019 \\ Vol. 4, Issue 3, ISSN No. 2455-2143, Pages 496-501 \\ Published Online July 2019 in IJEAST (http://www.ijeast.com)}

[2] J. M. Mahadi Abdul and A. M. Shukri, March 2015, "A Pilot Study: Development of Home Automation System via Raspberry Pi",International Conference on Biomedical Engineering (ICoBE).

[3] D Pavithra and B. Ranjith, April 2015, "IoT based Monitoring and Control System for Home Automation", Proceedings of 2015 Global Conference on Communication Technologies (GCCT).

[4] V. Sandeep, K. Lalith Gopal, S. Naveen, A. Amudhan and L. S. Kumar, August 2015, "Globally Accessible Machine Automation Using Raspberry Pi”, 2015 International Conference on Advances in Computing Communications and Informatics (ICACCI),

[5] Vamsikrishna Patchava, Hari Babu Kandala and P Ravi Babu, December 2015, “A Smart Home Automation Technique with Raspberry Pi using IoT ", International Conference on Smart Sensors and Systems (IC-SSS).

[6] Harsha S. L. S. Sri, Reddy C. S. and S Prince Mary, February 2017, "Enhanced Home Automation System using Internet of Things", International Conference on I-SMAC (IoT in Social, Mobile, Analytics and Cloud) (ISMAC).

[7] Kumawat Vikas, Jain Shubham, Vashisth Vikram, Mittal Neha and J. K. Bhupendra, April 2017, "Design of Controlling Home Appliance Remotely Using Raspberry pi ", International Conference for Convergence in Technology (I2CT).

[8] Quadri Syed Ali Imran and Sathish P, June 2017, "IoT Based Home Automation and Surveillance System", International Conference on Intelligent Computing and Control Systems (ICICCS).

[9] Chayapathy V , Anitha G. S and Sharath B, August 2017, "IOT BASED HOME AUTOMATION BY USING PERSONAL ASSISTANT”, International Conference On Smart Technologies For Smart Nation (SmartTechCon).

[10] Hussein Naser Abbas and mansoori Inas Al, September 2017, "Smart Door System for Home
Security Using Raspberry pi3”, 2017 International Conference on Computer and Applications (ICCA).

[11] Aktaruzzaman M. M, Badhan S. M, Adnan Sarwat, Alam M.R and Begum Most. T. A, December 2017, "Application of Cloudbridge Automation using Raspberry Pi”, 2017 IEEE Region 10 Humanitarian Technology Conference (R10HTC).

[12] Parthornratt Tussanai, Putthapipat Pasd, Kitsawat Dollachart and Koronjaruwat Prapap,July 2019, "A Smart Home Automation via Facebook Chatbot and Raspberry Pi", International Conference on Engineering Innovation (ICEI). 